\title{
Methodischer Zugang
}

Die Brauchbarkeit eines theoretischen Zugangs bemisst sich nicht nur an seiner Allgemeinheit und Auflösungsstärke beim Beschreiben sozialer Phänomene, sondern auch an seiner Übersetzbarkeit in ein methodisch instruktives Forschungsdesign. Die Frage, wie das Erkenntnisinteresse an der Entstehung einer organisierten Rechtsprechung und ihrer besonderen Binnenstrukturen operationalisiert werden kann, ist bislang unbeantwortet geblieben. Wie kann vor dem Hintergrund eines allgemeinen, systemtheoretischen Organisationsbegriffs und der genannten gesellschaftstheoretischen Prämissen eine abgesicherte Auswahl und Aufbereitung historischer Beobachtungen angestellt werden, die insbesondere Hinweise auf die Mitgliedschaftsfähigkeit dieser Erwartungen - und damit auf ihre formale Organisiertheit - liefert? Für die Beantwortung dieser Frage wird ein Zugang gewählt, der keiner standardisierten oder linearen Methode (etwa dem Schema Datensammlung, Datenanalyse, Datenaufbereitung) folgt.

Zur Darstellung dieses Vorgehens gehe ich in drei Schritten vor. In einem ersten Teil begründe ich die Wahl des methodischen Zugangs dieser Arbeit, bei dem die jüngere RKG-Forschung als historisch-empirisches Datenmaterial (Abschn. 4.1) dient. Zweitens stelle ich die wesentlichen Merkmale der dabei genutzten „funktionalen Methode“ (Luhmann 1962a, 1964) vor (Abschn. 4.2). In einem dritten Schritt zeige ich auf, wie sich aus der Fülle der historischen Forschungen konkrete Hinweise identifizieren lassen, die für eine Organisationsförmigkeit des RKG sprechen (Abschn. 4.3): Als Kriterium für die Auswahl von Ausdifferenzierungsmomenten einer organisierten Rechtsprechung untersuche ich dabei Erwartungskonflikte und Normbrüche. Schließlich leite ich aus dem gewählten theoretisch-methodischen Problemzugang zur Untersuchung der Organisationsförmigkeit des RKG die Gliederung des historisch-empirischen Teils ab.

R. Schwarting, Organisationsbildung und gesellschaftliche Differenzierung,

Organisation und Gesellschaft - Forschung,

https://doi.org/10.1007/978-3-658-32872-6_4 


\subsection{Jüngere RKG-Forschung als empirische Basis}

In einem allgemeinen Sinne lassen sich Methoden - ähnlich wie Theorien - als Normen der Absicherung wissenschaftlicher Kommunikation verstehen. Methoden geben Auskunft darüber, auf welche Weise Aussagen zustande kommen (vgl. Hirschauer 2008, 178). Entsprechend diesem sozialkonstruktivistischen Verhältnis von Theorie und Empirie ist bei der Untersuchung der Organisationsbildung des RKG und seiner gerichtsspezifischen Strukturen einzurechnen, dass der gewählte Forschungszugang den empirischen Gegenstand erst konstituiert (vgl. Kalthoff 2008, 17 f.; Hirschauer 2008, 169 ff.).

Zunächst zeigt sich bei der Erforschung des RKG eine für historische wie auch soziologische Studien ungewöhnlich hohe Fülle an Daten, wie sie erst ab dem 19. und 20. Jahrhundert zum Normalfall geworden ist (vgl. Abbott 2014; Blevins 2019). Neben den über 80.000 überlieferten Prozessakten werden in der RKG-Forschung Archivalien, Familienchroniken, persönliche Nachlässe oder gedruckte Materialien (wie Visitationsabschiede, Gemeine Bescheide oder die Kameralliteratur der RKG-Richter selbst) als Quellen genutzt.

Zum selektiven Umgang mit der enormen Menge an historischen Materialien zum RKG habe ich mich für die Auswertung der jüngeren RKG-Literatur und den in dieser verwendeten Primärdaten entschieden. Die Entscheidung gegen eine Primäranalyse zugunsten einer interpretativen Untersuchung war insbesondere durch den zeitlichen Rahmen des Projekts und die Grenzen der soziologischen Ausbildung bedingt. Eine eigene (rechts-)historische Quellenstudie über einen Fall in der Frühen Neuzeit setzt Erfahrungen in der Archivarbeit und ein Verständnis lateinischer und frühneuhochdeutscher Texte voraus. Sie erfordert die Fähigkeit zu einer historischen Kontextualisierung und verlangt damit eine entsprechende geschichtswissenschaftliche „Sonderqualifikation“ (Lepsius 1976, 122; vgl. auch Schützeichel 2004, 14 ff.).

Ein kontrollierender Abgleich zwischen den Quellen und ihrer Auswertung war im Rahmen dieser Arbeit auch angesichts des Umfangs der RKG-Forschung und der Vielfalt der darin behandelten Gegenstände und Materialien nicht leistbar. Der empirische Teil vermag demnach keine Einblicke in die historische Realität zu geben. Für nomologisch-deduktive oder historiografische Forschungen wird die hier vorgelegte Untersuchung insofern wenig informativ sein. Soziologen können allerdings bei der Verwendung bereits entdeckter Quellen und Literatur ,sicher sein, wenn sie von Theorie ausgehen, in den Quellen ganz andere Dinge zu entdecken, als den Historikern auffallen würden“ (Luhmann 1994b, 260; siehe auch Stichweh 1998, 70). Weber formuliert in diesem Zusammenhang, dass sich ein ,solches vergleichendes Übergreifen auf andere Fachgebiete“ (Weber 2016 
[1920], 119) für eine historisch interessierte Soziologie nicht vermeiden lasse, und dass diese gleichwohl ihrer Absicht nach empirisch orientiert ist.

Bildlich gesprochen bedarf es für eine ergebnisoffene Materialauswahl aus dem geschichtswissenschaftlichen Forschungsbestand einer Methode, die sich weniger mit einer Landkarte als mit einem Kompass vergleichen lässt. Der erste Kontakt mit der Literatur der historischen Forschungen zum RKG ähnelte dabei einer problemorientierten Erkundung von Unwahrscheinlichkeiten (vgl. Luhmann 2005, 26; siehe auch Stolleis 2008, 28; Hirschauer 2008, 175) im Sinne einer , archäologischen Spurensicherung' (vgl. Ginzburg 2011). Archäologisch war die Sichtung und Auswahl der historischen RKG-Forschung deshalb, weil zwischen der hier vorgelegten Arbeit und ihrem Gegenstand mehrere Jahrhunderte liegen. Bei diesem Rückgriff handelt es sich um einen Zugang, der weder einer standardisierten Methodik der empirischen Sozialforschung folgt noch einer einheitlichen historischen Methode zuzurechnen ist. Im Unterschied zu einer standardisierten Methode ist die herangezogene Theorie keine ,Optik', die nur bestätigt, was zuvor hineinerfunden wurde, sondern ein konzeptioneller Begriffs- und Beobachtungsrahmen zur intersubjektiv nachvollziehbaren Ableitung empirischer Aussagen (vgl. Hirschauer 2008, 176 ff.; Kalthoff 2008, 21 ff.).

Leitend waren in dieser Arbeit dabei zwei methodische Maximen, die an ethnografische Forschungen angelehnt sind. Zunächst betrachte ich die geschichtswissenschaftliche Literatur einerseits distanziert und andererseits aus der Nähe: Eine distanzierte Haltung nehme ich gegenüber den Begrifflichkeiten und Narrationen der historischen Forschung ein; eine Annäherung erfolgte dagegen bezogen auf die einzelnen Situationsbeschreibungen über die Bedingungen der Rechtsprechung am RKG. Geeint werden beide Haltungen durch den Gebrauch der vorgestellten theoretischen Konzepte (siehe Kap. 3). Der Anspruch bei der Selektion und Reinterpretation der RKG-Forschungen war zudem, in sozialer, sachlicher und zeitlicher Hinsicht möglichst heterogene Beiträge über Prozessakten, Verhörprotokolle oder zeitgenössische Schriften einzubeziehen. Diese dienen gleichsam als Verhaltensdokumente für die Analyse von Rollenauffassungen, Situationsauslegungen und Selbstdarstellungen (siehe dazu weiter in Abschn. 4.2).

Hinsichtlich der Frage nach dem Verhältnis von Theorie und Empirie stellen sich mithin methodologische Probleme historischer Komparatistik, die in der historischen Soziologie weitgehend „ungelöst“ (Schützeichel 2009, 279; siehe auch Schützeichel 2004, 13) geblieben sind. Während der Theoriebezug bei singularisierenden Zugängen zu gering ausfällt, stellt sich dieser im Fall universalisierender Ansätze als zu dominant heraus (vgl. Welskopp 1995, 353 ff.; 2015a, 189 ff.; siehe auch Hacke/Pohlig 2008; Pohlig 2013). Mit der gewählten Fragestellung nach der Organisationsbildung des RKG und den Strukturausprägungen 
einer organisierten Rechtsprechung ist verbunden, dass die ausgewählten (rechts-) historischen RKG-Forschungen anhand der zuvor eingeführten Unterscheidungen selegiert und reformuliert werden müssen. Wie oben erläutert, liegt die Funktion von Theorie darin, Kriterien im Sinne von Begriffs- und Beobachtungsmitteln für das Abstecken von Empirie zu liefern (vgl. Kalthoff 2008). Die entwickelten Unterscheidungen werden dabei nicht einfach wie eine Beweisführung auf einen Fall angewandt. Vielmehr ist der Anspruch, theoretische und empirische Beobachtungen problem- und gegenstandsorientiert ins Verhältnis zu setzen. Die Theorie orientiert dabei das empirische Suchverhalten (vgl. Luhmann 2017, 87). Sie gewährleistet auch, dass man die Einsichten aus der geschichtswissenschaftlichen Literatur von der jeweiligen Erkenntnisabsicht abgrenzen und für die eigene Problemstellung rekonstruieren kann (vgl. Stichweh 1998, 70). Denn wonach sollte man - wie hier bei der Genese einer Organisation in der Vormoderne - suchen, ohne im Vorfeld einen bestimmten Begriff von Ausdifferenzierung, Organisation, Recht und Gesellschaft entwickelt zu haben (siehe Kap. 3; siehe auch Stollberg-Rilinger 2012, 290). ${ }^{1}$

Wenn man sich gegen die historische Narration als Darstellungsform entscheidet, sondern bereits für ,fremde " Forschungsinteressen und Disziplinen erstellte Texte auf Hinweise zu einer eigenen Fragestellung hin abtastet, sind dafür besondere Unterscheidungs- und Übersetzungsleistungen erforderlich: Eine zentrale Herausforderung besteht darin, dass die Forschungsliteratur - sowie die dabei genutzten Quellen - vorgefilterte Beschreibungen und Narrative mitführen, die im vorliegenden Fall mit (rechts-)historischen Deutungen sowie zeitgenössischen Sinnbezügen überlagert sind. Den geschichtswissenschaftlichen Forschungen ist gemeinsam, dass es sich bei ihnen um fragmentarische Fallschilderungen und Kontextualisierungen handelt (vgl. Stolleis 2008, 26 f.). Je nach Fragestellung, Quelle, Methode und theoretischem Zugang werden unterschiedliche Aspekte betont, die weder konsistent ineinandergreifen noch anhand der hier verfolgten Problemstellung kohärent sortiert sind, sondern sich teilweise widersprechen. Der Aussagewert des ausgewählten Materials für die hier verfolgte Frage nach der Organisationsförmigkeit des RKG fällt entsprechend unterschiedlich aus. Die Beobachtungen der RKG-Forschung galt es vor diesem Hintergrund zu dekomponieren. Das Anfertigen interpretativer Fremdbeschreibungen entwickelte sich dabei als ein iterativer Forschungsprozess, bei dem die unterschiedlichen Beiträge und Materialien schrittweise aufeinander bezogen wurden. Das aus der

\footnotetext{
${ }^{1}$ Umso herausfordernder ist es für die soziologische Auswertung, wenn in historischen Studien ,moderne“ Termini verwendet werden (z.B. Dienstleistungen, Regierung, Gesetz), ohne dass deutlich wird, inwiefern es sich dabei um Quellenbegriffe handelt oder bereits um ein theoretisches Konzept.
} 
Sichtung der RKG-Literatur hervorgegangene Vorverständnis wurde durch allgemeinere historische Forschungen über die gesellschaftlichen Strukturbedingungen im Alten Reich weiter erschlossen und angereichert. Dieses Vorgehen ermöglichte es, gesellschaftliche und gerichtsspezifische Beschreibungen bzw. die darin enthaltenen Deutungen vergleichend zu reanalysieren und dabei insbesondere offen für unvorhergesehene empirische Entdeckungen zu bleiben.

Insgesamt lassen sich das hier skizzierte Güteproblem (der nichtsystematischen Abgleichung der Quellen mit den historischen Forschungsaussagen), das Fragmentierungsproblem (der zwangsläufig unvollständigen Interpretation der RKG-Forschungen) sowie die damit verbundenen blinden Flecke nicht vollständig beheben. Sie können durch die Wahl eines weiteren alternativen Forschungsstandpunktes - als einem Beobachter dritter Ordnung - im Rahmen von Anschlussforschungen aufgegriffen werden.

\subsection{Funktionale Methode}

Um aus den historischen RKG-Forschungen Hinweise zu identifizieren, die Aufschluss über die Vermutung einer Organisationsbildung in einer ständischen Gesellschaft geben können, diente in dieser Arbeit die sogenannte funktionale Methode als Anleitung (vgl. Luhmann 1962a, 617-644; 1964, 1-25; 1999a, 17 ff.; 2010, 19-28). Bezeichnet ist damit die systemtheoretische Methodologie hinter Luhmanns Gesellschaftstheorie. Die funktionale Analyse ist dabei vor allem eine interpretative Methode. Konkret handelt es sich um eine funktionalistische Variante qualitativ-empirischer Sozialforschung, die weniger zur Neutralität als zur methodischen Einrechnung der eigenen Forschungsperspektive und der Kontrolle dieses Standpunktes zwingt (vgl. Nassehi 2008, 98). Sie kann als ethnomethodologisch verstanden werden, insofern sie ,sich für die Selbstentfaltung von Situationen interessiert" (ebd., 99) und sich um das Aufzeigen einer auch anders möglichen Selektionsgeschichte bemüht. Sie unterscheidet sich gleichwohl von ethnomethodologischen und ethnografischen Zugängen dadurch, dass sie weniger auf den „engen Kontext des Beobachtbaren“ beschränkt ist, sondern zusätzlich „mit einer Gesellschaft rechnet, in der diese Kontexte vorkommen“ (ebd., 100).

Der Vorzug der funktionalen Methode kann gegenüber individualisierenden oder universalisierenden Zugängen auch darin gesehen werden, dass sie durch ihre Einbettung in eine soziologische Gesellschaftstheorie die gewonnenen Beobachtungen nicht von systematischen Generalisierungen trennt. Anschlussfähige 
Vergleiche können potenziell in diachroner und synchroner Hinsicht vorgenommen werden, ohne den empirischen Einzelfall zu ignorieren. Nach Rudolf Stichweh gibt es zwar ,keinen Grund anzunehmen, daß Systemtheorie und funktionale Analysen im besonderen Grade Schwierigkeiten mit historischen Fragestellungen hätten“ (1998, 71). Jedoch werden funktionale Differenzierungstheorie und historische Analysen oft als inkompatibel verstanden (z. B. Sewell 2005; Oexle 1991). Dazu mag beigetragen haben, dass innerhalb der US-amerikanischen Organisationsforschung die Begriffe funktional und funktionalistisch nicht selten mit deterministisch gleichgesetzt werden (z. B. bei Bucheli/Wadhwani 2014, 9). Die funktionale Methode unterscheidet sich gleichwohl vom klassischen Funktionalismus dadurch, dass die betrachteten Probleme der Erwartungsbildung und deren Lösungen nicht normativ vorgegeben sind, sondern Vorhandenes als kontingent und Verschiedenartiges als vergleichbar aufgefasst werden. Im Unterschied zu Talcott Parsons' Strukturfunktionalismus hat Luhmann in seiner Systemtheorie die Strukturkomponente der Funktionskomponente untergeordnet (vgl. 1984, 83 ff., 379-387; 2010, 26; 2013, 47 ff.). Gemäß dieser theoretischen Umstellung werden soziale Problem-Lösung-Konstellationen nicht vorempirisch postuliert. In Abgrenzung zu einem probabilistischen Kausalitätsbegriff vertritt Luhmann ein possibilistisches Funktionsverständnis, das danach fragt, welche Strukturen funktional für die Stabilisierung bestimmter Verhaltenserwartungen sind. Funktionen stellen dabei äquivalente Problemlösungen dar, die zugleich den Blick für alternative, auch anders mögliche Lösungen schärfen (vgl. Luhmann 1962a, 617 ff.; 1964, 4 ff.). Rudolf Stichweh bezeichnet den „Analysestil der Systemtheorie“, mit der die Theorieanlage empiriefähig gemacht wird, denn auch als ,implizit historisch“: „Man betrachtet zu einem gegebenen Zeitpunkt ein Spektrum von entstandenen Alternativen, versucht die Bedingungen herauszufinden, die einzelne dieser Alternativen gegenüber anderen begünstigen und systematisiert diese Erfahrungen“" (Stichweh 1998, 77).

Worauf beruht die funktionale Methode im Einzelnen? Der Ausgangspunkt dieses Zugangs besteht im Wesentlichen darin, ein Bezugsproblem zu identifizieren, das einerseits theoretisch begründet wird und andererseits aus dem empirischen Kontext selbst entnommen ist. Die Wahl eines Bezugsproblems entspricht der Angabe einer System- bzw. Strukturreferenz (vgl. Luhmann 2011, 4, 26). Mit der theoretisch abgeleiteten Bestimmung eines Bezugsproblems wird dabei ein Bereich abgesteckt, der zur Identifikation von Verhaltenserwartungen dient, die als Selektionsvorgänge den Strukturaufbau bei der Ausdifferenzierung von Systemen leisten. Bei der Formulierung von Bezugsproblemen bzw. Bezugssystemen handelt es sich deshalb um eine Abstraktionsleistung, bei der Verschiedenartiges als gleich im Sinne von funktional äquivalent behandelt wird (vgl. 
Luhmann 1964, 8). Damit erklären Bezugsprobleme nicht das faktische Vorkommen bestimmter funktionaler Leistungen, sondern verweisen wie angesprochen auf andere Möglichkeiten. Das Bezugsproblem dient dabei als eine Art invarianter Kontext für den Einbezug möglicher Problemlösungen und erzeugt eine Problemstufenordnung von Ermöglichungsbedingungen und erwartungsbasierten Verweisungszusammenhängen (vgl. Luhmann 1964, 24 f.).

Wichtig für das Verständnis der Problemstufenordnung ist, dass Funktionalität keine Steigerbarkeit vorsieht. Aussagen, nach denen die Problemlösung A funktionaler als B ist, sind äquivalenzfunktionalistisch nicht gemeint. A ist nicht weniger funktional als B, sondern wenn funktional, dann funktional äquivalent (vgl. Luhmann 1984, 85 ff.). Mittels der Rekonstruktion von Problemstufenordnungen lassen sich nicht nur funktional äquivalente Lösungen mit Blick auf ihre Substituierbarkeit beobachten, sondern zugleich auch spezielle Folgeprobleme mit latenten, dysfunktionalen oder brauchbar-illegalen (Luhmann 1999a, 304 ff.) Problemlösungen. ${ }^{2}$ Diese Folgeprobleme können dann wiederum als Bezugsprobleme für weitere funktionale Analysen betrachtet werden. Denn: Dass funktional äquivalente Lösungen eines gemeinsamen Bezugsproblems in einen Zusammenhang gebracht werden können, heißt nicht, dass damit Probleme eindeutig gelöst sind. Diese Relationierung von Problem und Problemlösung ermöglicht es ,lediglich', kommunikative Ereignisse als Informationen eines sozialen Systems aufzufassen und dadurch in Beziehung zu setzen (vgl. Luhmann 1962a, 617; 1964, 19; 1999a, 23).

Luhmann veranschaulicht die funktionale Methode am Beispiel von Statuserwartungen. Zunächst unvergleichbare Einrichtungen, wie eine Sekretariatsstelle und ein Dienstwagen, können neben ihrem (manifesten) Zweck auch eine dekorative (latente) Funktion übernehmen. Beide können die Distanz, Überlegenheit und Entscheidungsfreiheit eines Ranges symbolisieren (vgl. Luhmann 1966a, 99). Ein anderes Beispiel sind Rollenkonflikte. Jede soziale Ordnung ist mit dem Problem konfrontiert, verschiedene Rollen vorsehen und diese miteinander verbinden zu müssen. Das Problem konfligierender Rollen kann durch die Institutionalisierung von Hierarchie gelöst werden, etwa wenn der Situationsdefinition des Ältesten einer Gruppe auch von Dritten Priorität zugesprochen wird. Rollenkonflikte lassen sich gleichwohl auch zeitlich entschärfen, wenn Interaktionspartner räumlich

\footnotetext{
${ }^{2}$ Latenz wird hier nicht im Sinne von verborgen, unterbewusst, nichtwissend oder als fehlende Absicht verstanden, sondern als Inkommunikabilität ,mit der Funktion des Strukturschutzes“ (Luhmann 1984, 459); latent sind also Erwartungen, die „,nicht offen zum Ausdruck“ (1999a, 359) gebracht werden bzw. nicht manifest thematisierbar sind, ohne Erwartungsgrenzen offenzulegen (siehe auch Merton 1940).
} 
isoliert werden oder ein Dritter zur Entscheidung des Konflikts akzeptiert wird (vgl. ebd.).

Bei den genannten Beispielen wird deutlich, dass sich Systemprobleme letztlich auf Probleme der Erwartungsstabilisierung zurückführen lassen; und dies ganz unabhängig von ihrer Typik - sei es als Interaktion, als Organisation oder Gesellschaft (siehe Abschn. 3.1). Erwartungsgrenzen verhindern dabei, dass Kommunikationen , ungeprüft ‘ die Beziehungen zu anderen Teilen der Umwelt färben. Erst die Orientierung an Systemstrukturen ermöglicht mithin die Ausbildung relativ verlässlicher Verhaltenserwartungen in Systemen. Nimmt man an, dass soziale Systeme ihre Grenzen normativ stabilisieren, auch wenn faktisch von bestimmten systembezogenen Erwartungen abgewichen wird, so bedarf es auch Problemlösungen für den Umgang mit Enttäuschungen. Als funktional äquivalente Abwicklungsmechanismen für den Umgang mit derartigen Folgeproblemen beschreibt Luhmann die Möglichkeit der Anpassung von Erwartung an die Wirklichkeit, also ein „Lernen“, beispielsweise in Form von Rechtfertigungen und Entschuldigungen (vgl. Luhmann 1972a, 53 ff.; 1984, 217 ff.).

Als das Bezugsproblem formaler Organisation benennt Luhmann in seinem Werk „Funktionen und Folgen formaler Organisation“ die Grenzziehung bzw. das Invarianthalten von Verhaltenserwartungen in einer veränderlichen Umwelt (vgl. 1999a, 24 f.). Für die Herstellung von Erwartungssicherheit lassen sich neben Organisationsbildung ebenso alternative und ergänzende Mechanismen analysieren, wie das Vertrauen in Personen (vgl. Luhmann 2009 [1968]), die Setzung von Rechtsnormen oder die Verpflichtung auf bestimmte Werte (vgl. Luhmann 1972a, 217 ff.). Mit Blick auf das hier gewählte Interesse an der historischen Entstehung von Organisationen werden diese Formen der Erwartungsstabilisierung in der Analyse an empirisch relevanten Stellen einbezogen. Der Unterschied zwischen Recht, Personenvertrauen und Organisation wird gleichwohl darin gesehen, dass diese Phänomene verschiedene Institutionalisierungs- und Bindungsgrade in der Ausbildung von Erwartungserwartungen aufweisen. Für Organisationen ist dabei spezifisch, dass sie kooperative Verhaltenserwartungen für ihre Mitglieder auf der Basis von Geld in generalisierter Form - also situationsübergreifend - motivieren und konditionieren.

Mit der Annahme, dass es sich beim RKG um eine Organisationsbildung handelt, formuliere ich schließlich als Bezugsproblem die Frage, welche spezifischen (Teil-)Strukturen bei der Rechtsprechung am Gericht gegenüber der gesellschaftlichen Umwelt als invariant verstanden wurden. Konkret frage ich danach, welche Situationsauslegungen und Rollenauffassungen einen Beitrag zum Aufbau einer gerichtseigenen Erwartungsordnung und, damit verbunden, zur Differenzierung 
von Gerichtsorganisation und Gesellschaft leisteten. Mit anderen Worten: Welchen Erwartungen wurde personen- und statusübergreifend eine hervorgehobene Geltung zugesprochen und mit einer organisierten Rechtsprechung in Verbindung gebracht?

\subsection{Erwartungskonflikte als Indiz für Ausdifferenzierungen}

Die funktionale Methode sieht sich also ihren Gegenstand als eine Lösung an, bezieht diese Lösung auf (theoretisch abgeleitete) systemrelative Probleme und entdeckt dabei Alternativen auf beiden Seiten. Weiterführend stellt sich die Frage, wie innerhalb der RKG-Forschung eine Auswahl von Momenten der Grenzziehung unterschiedlicher Erwartungsordnungen bzw. konkret der Trennung einer organisierten Rechtsprechung am RKG gegenüber gesellschaftlichen Rechtserwartungen beobachtbar ist. Im theoretischen Teil wurde ein Verständnis von Systemgrenzen entwickelt, das sich an konfligierenden Erwartungen orientiert. Als Erwartungskonflikte verstehe ich divergierende Situationsauffassungen und Rollendefinitionen, bei denen gerichtlich und reichsrechtlich gesetzten Verhaltensanforderungen gegenüber gesellschaftlich tradierten Erwartungen ein Monopol auf Legitimität zugesprochen (oder ein solches unterlaufen) wurde.

Grundlage für diesen Zugang ist die Prämisse, dass die Ausbildung der Systemgrenzen zwischen Gesellschaft und Organisation, wie im theoretischen Teil erläutert, auf der Trennung von gesellschaftlichen Normen und formalen Mitgliedschaftserwartungen einschließlich der damit verbundenen informalen Erwartungen basiert. Normbrüche und Erwartungskonflikte werden dabei nicht als Abweichungen von Rationalitätsansprüchen, sondern als (dys-)funktionale Lösungen für spezifische Systemprobleme verstanden.

Weil Systemgrenzen zugleich Ausdruck von Erwartungsgrenzen sind, geben Erwartungskonflikte Anhaltspunkte für instabile Normen und die Differenzierung unterschiedlicher Verhaltenserwartungen. In loser Anlehnung an ethnomethodologische Zugänge nutze ich in dieser Arbeit deshalb Erwartungskonflikte als empirische Referenzpunkte für die Identifikation sozialer Systembildung. Die damit verbundene Suche nach divergierenden Rollenauffassungen und Situationsdefinitionen ist insofern methodisch instruktiv, weil Konflikte Aufmerksamkeit auf sich ziehen und Ressourcen an sich binden. Konflikte sind damit kommunikativ greifbar (vgl. Luhmann 1972a, 53 ff.; 1999a, 40ff; siehe auch Garfinkel 1967, 35 ff.; Silverman 1993). 
Ein systemtheoretischer Konfliktbegriff ist dabei kommunikationstheoretisch ausformuliert und lässt sich gerade deshalb auf der Ebene von sowohl Organisation als auch Interaktion verstehen. In Luhmanns Werk wird der Begriff zunächst interaktionsnah eingeführt (vgl. Luhmann 1999a, 55 f., 100; Lieckweg/Wehrsig 2001, 51; Kieserling 1999, 267): Von Konflikten lässt sich ,immer dann sprechen, wenn ein Teilnehmer an Interaktionen es ablehnt, Selektionsvorschläge zu übernehmen und diese Ablehnung mitteilt" (Luhmann 1975a, 16; 2018, 22, Herv. i. O.). Sie entstehen also, wenn die Ablehnung einer Kommunikation - das Nein zum Thema der weiteren Kommunikation wird. Ein einfaches Nein ist dabei noch kein Konflikt, sondern erst die doppelte Verneinung, beispielsweise im Sinne von „Nie meldest du dich zurück“. Konflikte entsprechen abstrakt formuliert damit der operativen Verselbständigung eines Widerspruchs (vgl. Luhmann 1980b, 99 ff.; 1984, 530; siehe auch Nollmann 1997, 104).

Aufgrund ihres relativ hohen Aufmerksamkeitsniveaus und ihres Ressourcenverbrauchs verlangen Konflikte nach einer Konditionierung und einem Deutungsmonopol für die Auslegung der Situation. Im Unterschied zu Interaktionen, in denen Konflikte als Thema kaum Alternativen finden und die Kommunikation überziehen, sind Konflikte in Organisationen mit ihrer internen Differenzierung typischerweise verteilt. Sie können an einer Stelle weiterlaufen, ohne andere Bereiche zu tangieren. Wie oben eingeführt, markiert die Mitgliedsrolle eine Konfliktschwelle, die angibt, welche Konflikte hierarchisch entscheidbar sind (siehe Abschn. 3.2). Offene Abweichungen von der formalen Erwartungsordnung verlangen damit in der Regel nach einer expliziten Entscheidung seitens der Hierarchie. In einem zweiten Schritt kann dann danach gefragt werden, ob und inwiefern die faktischen Rollenkonflikte und Normbrüche als funktional oder dysfunktional für das jeweilige Gesamtsystem angesehen werden und welche Verhaltenserwartungen damit verbundene Systemprobleme lösen (vgl. Luhmann 1984, 530 ff.; 1999a, 304 ff.). „Welche Systemgrenze in der Lage ist, ihre Erwartungen im System zu normieren, ist dabei eine Machtfrage und nie in einem absolut einseitigen Sinne zu lösen“ (Luhmann 1966a, 81). Erst von diesem Standpunkt aus kann untersucht werden, ob ein System - wie hier Organisationen - normatives Eigengewicht gewinnt und als solches eine eigene Systemrationalität ${ }^{3}$ entwickelt (vgl. Luhmann 1966a, 64 f.).

\footnotetext{
${ }^{3}$ Zum Begriff der Systemrationalität und der Abgrenzung zu (normativen) Zweckbegriffen siehe Luhmanns gleichnamigen Band „Zweckbegriff und Systemrationalität“ (1973; siehe auch 1971).
} 
Die Suche nach divergierenden Rollendefinitionen und Situationsdeutungen ist damit der methodische Schlüssel für die Analyse von Momenten der Ausdifferenzierung einer organisierten Rechtsprechung am RKG. Entsprechend habe ich die RKG-Forschung danach ausgewertet, inwiefern die darin formulierten Beobachtungen Anhaltspunkte für organisatorische Normalerwartungen und Selbstverständlichkeiten widerspiegeln und welche Verhaltensschilderungen dagegen Hinweise auf enttäuschte und begründungsbedürftige Erwartungen geben. Erwartungskonflikte können dabei in zweierlei Hinsicht als Indizien für die Ausbildung organisatorischer Grenzziehungen genutzt werden: einerseits für die Differenzierung von Organisationen gegenüber ihren gesellschaftlichen Umwelten sowie andererseits für eine Trennung von formalen und informalen Strukturen innerhalb von Organisationen.

Über die Ausdifferenzierung gesellschaftlicher und gerichtlicher Teilstrukturen geben die einschlägigen Beiträge aus der historischen Literatur in unterschiedlichem Maße Aufschluss. Die historische Distanz zum Gegenstand erweist sich nicht nur als Einschränkung, sondern auch als Vorteil. Der besondere ,Quellenwert ' der RKG-Forschungen besteht für eine historisch-soziologische Organisationsanalyse nicht zuletzt in der empirischen Reichhaltigkeit der überlieferten Materialien, die damit bereits aus vielfältigen Perspektiven auf den Gegenstand erschlossen wurde (siehe Abschn. 2.2).

Letztlich standen im Verlauf der explorativen Auseinandersetzung mit der historischen Literatur insbesondere folgende Fragen im Vordergrund: Welche sozialen Unterscheidungen wurden zwischen Personen gemacht und welche Statuszuweisungen waren damit verbunden? Welchen Erwartungen verpflichteten sich die verschiedenen Mitglieder des Gerichts? Welchen Erwartungen wurde im Konfliktfall durch welches Verhalten Vorzug geleistet bzw. wie wurden Geltungsansprüche zurückgewiesen? Welchen gesellschaftlichen Problemen sollte durch die Trennung von Verhaltenserwartungen Rechnung getragen werden?

Im Einzelnen gliedert sich die Auswertung und Aufbereitung der RKGForschungen nach vier thematischen Fragekomplexen, die entsprechend die Abschnitte der Fallstudie bilden.

\section{1) Formale Strukturausprägungen organisierter Rechtsprechung}

Inwiefern wurde die Rechtsprechung am RKG durch eine formale Erwartungsordnung strukturiert und diese gegenüber gesellschaftlichen Erwartungen invariant gehalten? Was waren die Inhalte der Formalstruktur? In Bezug auf welche Referenzen wurde die Formalstruktur formuliert und angepasst? (Abschn. 6.1) 


\section{2) Informale Strukturausprägungen einer organisierten Rechtsprechung}

Welche informalen Erwartungen bildeten sich bei der Rechtsprechung am RKG als Problemlösungen aus? Welche informalen Kontaktsysteme richteten sich im Verfahrensalltag zwischen dem Gerichtspersonal und den Streitparteien ein? (Abschn. 6.2)

3) Formale Strukturen im Besonderen: Personal, hierarchische Kommunikationswege und Entscheidungsprogramme

Inwiefern orientierte sich das Verhalten der RKG-Mitglieder an formalen Mitgliedschaftserwartungen? Durch welche Strukturen waren Auswahl-, Beförderungs- und Entlassungsentscheidungen bedingt? Welche formalen Kommunikationswege hatten die Mitglieder und Nichtmitglieder zu beachten? Inwiefern richtete sich die Rechtsprechung nach programmatischen Richtigkeitsbedingungen und durch welche Regeln wurden (prozess-) rechtliche Entscheidungen strukturiert? (Abschn. 6.3)

4) Formale Strukturen im Besonderen: Außendarstellung

Inwiefern lässt sich für das RKG eine exponierte Außendarstellung festmachen? An welchen Verhaltenserwartungen orientierte sich die Darstellung des Gerichts für Nichtmitglieder? Inwiefern lassen sich Verhaltenserwartungen identifizieren, die nicht Gegenstand einer offiziellen Darstellung des RKG waren und die nach außen gegenüber Dritten kommunikativ abgetrennt wurden? (Abschn. 6.4)

Open Access Dieses Kapitel wird unter der Creative Commons Namensnennung 4.0 International Lizenz (http://creativecommons.org/licenses/by/4.0/deed.de) veröffentlicht, welche die Nutzung, Vervielfältigung, Bearbeitung, Verbreitung und Wiedergabe in jeglichem Medium und Format erlaubt, sofern Sie den/die ursprünglichen Autor(en) und die Quelle ordnungsgemäß nennen, einen Link zur Creative Commons Lizenz beifügen und angeben, ob Änderungen vorgenommen wurden.

Die in diesem Kapitel enthaltenen Bilder und sonstiges Drittmaterial unterliegen ebenfalls der genannten Creative Commons Lizenz, sofern sich aus der Abbildungslegende nichts anderes ergibt. Sofern das betreffende Material nicht unter der genannten Creative Commons Lizenz steht und die betreffende Handlung nicht nach gesetzlichen Vorschriften erlaubt ist, ist für die oben aufgeführten Weiterverwendungen des Materials die Einwilligung des jeweiligen Rechteinhabers einzuholen. 